\title{
Guidance System Based on Image Processing
}

\author{
Dahai Yu and Manman Shen \\ School of electrical information of Changchun Guanghua University, Changchun, China, 130031
}

\begin{abstract}
To solve the problem of blind travel, a guide system based on image processing is designed. The visual information collected by a CCD camera. The voice information is used to communicate with the user. The overall design of this system is given. Recognize the zebra line by the bipolar system value. Recognize the blind road using saturation histogram and Gaussian function.
\end{abstract}

Keywords-blind identification; zebra crossing recognition; image processing; guide

\section{INTRODUCTION}

According to the World Health Organization (WHO) statistics in 2010, the total number of people in the world with vision impairment is estimated to be 285 million. 39 million are whole blind. While China has the most people, the number of blind people is also the most. There is about 5 million blind people in China. Due to the physiological defects and the increasingly complex living environment, it brings many inconveniences to the blind people's life. In view of the inconvenience of blind people, guide dog and guide stick gradually become tools to help blind people travel. However, the guide dog is not easy to train and the cost is high, so the detection range of the blind guide rod is limited.

$\mathrm{H}$ Wang has designed an interactive guide robot. The robot is composed of haptic device and human-computer interaction system. Analyze the 2D information through the haptic system and transmits the information to the user. But the robot can only detect the surrounding obstacles, not the main effective identification the traffic signs. Zhang Ying designed a guide robot based on embedded technology to identify obstacles and traffic signs. But the robot will not receive the voice message to the blind, which caused a lot of inconvenience to the blind. Han Xuefeng designed an interactive guide robot, through the sensor detection of the external environment, and transfer in the form of voice for the blind. But the robot of traffic sign recognition effect is poor, which can't meet the actual needs of the blind. Zhang Zhimei et al designed a crawler type guide robot. Use ultrasonic sensor to track the trajectory of black ground preset to avoid obstacles. But it can't work in no-black environment.

In view of the deficiency of the blind guiding technology at present, the design of intelligent guide system has great practical significance.

\section{STRUCTURE OF GUIDANCE SYSTEM}

According to the restriction of the activity of the blind, the function of the guide robot is confirmed, and the overall scheme of the guide system is worked out. As shown in Figure I.

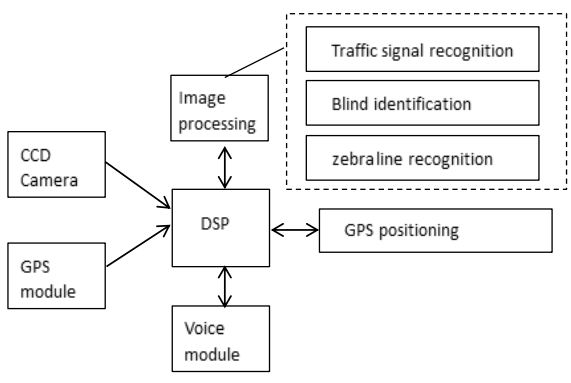

FIGURE I. OVERALL SCHEME DESIGN

A camera is used to collect the image information of the environment. The data are transferred to DSP. GPS module is used to position the user. Voice module is used to receive or play voices for users. The data are also transferred to DSP. Do some calculate in DSP including traffic signal recognition, Blind identification, zebra crossing recognition and so on.

\section{ZEBRA LINE RECOGNITION}

The zebra belt consists of a group of parallel strips with alternate black and white intervals. The difference of color between black and white stripes is very obvious. Gray contrast is very strong. The rule of black and white alternating is strong. So we can use the bipolar coefficient of the image to characterize and quantify the intensity of the intensity contrast of the zebra line region. Analyze and judge the region with strong gray contrast in the road image by the bipolar system value. If the test area is in the zebra line region, the value of the bipolar system is very high. Otherwise, if the region to be inspected is not a zebra region, its gray value is basically the same, and the value of the bipolar systemis small.

First we can use a threshold to segment the image.

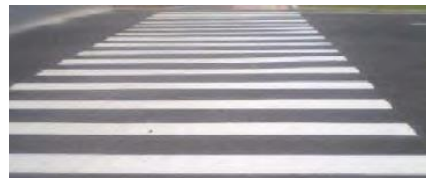

(a) ORIGIN IMAGE

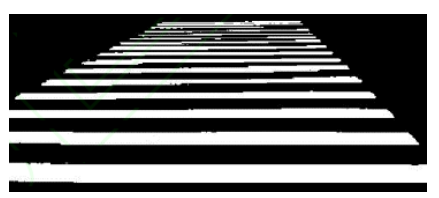

(b) THRESHOLD SEGMENTATION

FIGURE II. IMAGE SEGMENTATION

Then suppose $\left(\mu_{1}, \sigma_{1}\right)$ is the average value of density distribution function of black pixels. $\left(\mu_{2}, \sigma_{2}\right)$ is the average 
value of density distribution function of whith pixels. $n_{1}, n_{2}$ presents the pixel number of black and white.

$$
\alpha=\frac{\mathrm{n}_{1}}{\mathrm{n}_{1}+\mathrm{n}_{2}}
$$

$$
\sigma_{0}^{2}=\alpha \sigma_{1}^{2}+(1-\alpha) \sigma_{2}^{2}+a(1-\alpha)\left(\mu_{1}-\mu_{2}\right)^{2}
$$

The bipolar coefficient $\gamma$ is

$$
\gamma=\frac{1}{\sigma_{0}^{2}}\left[\alpha(1-\alpha)\left(\mu_{1}-\mu_{2}\right)^{2}\right]
$$

$\gamma$ is a value between 0 and 1 . When $\gamma$ is 0 , the image is not zebra area. And when $\gamma$ is 1 , the image must be zebra area.

Then search the edge of the zebra. When there are parallel lines in the image. It could be recognized as zebra lines.

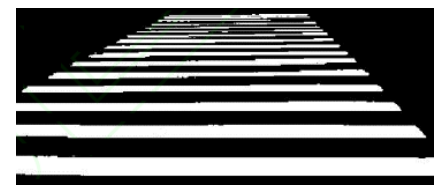

(a) THRESHOLD SEGMENTATION

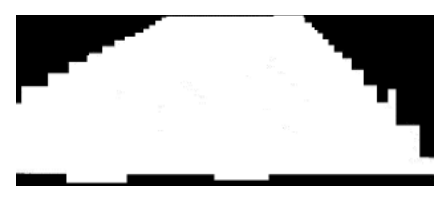

(b) REGIONS USING BIPOLARITY

FIGURE III. RESULT OF BIPOLAR COEFFICIENT

At the end, use the edge of the zebra line. Then we can get the zebra lines info rmation.

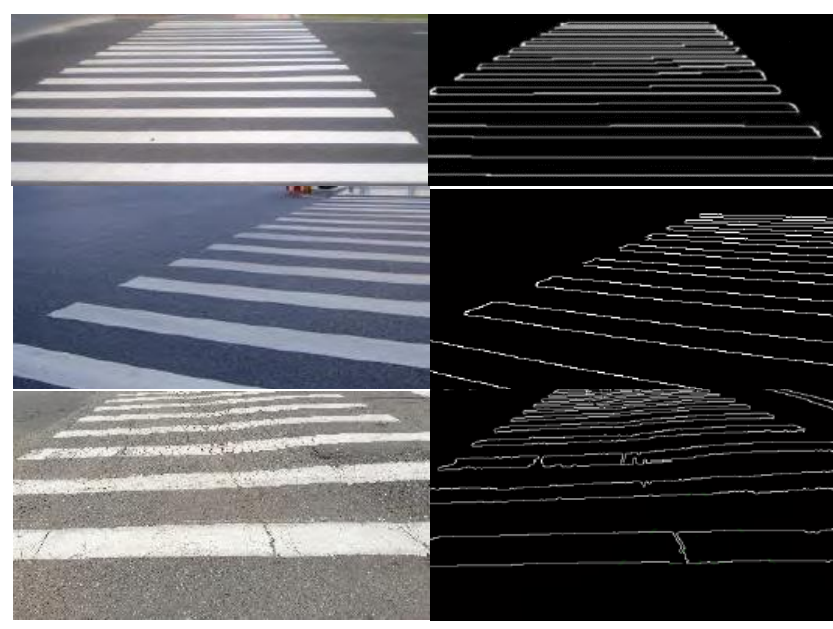

FIGURE IV. RESULT OF ZEBRA CROSSING RECOGNITION

\section{BLIND ROAD RECOGNITION}

The color of b lind road is usually very bright. So the colors can be used to detect the characteristics of the blind road. This paper gets the blind area using image segmentation. Extract the edge of blind road. First, convert the image from RGB to
HSI color space. HSI has three color components. Use the saturation histogram to segment image.

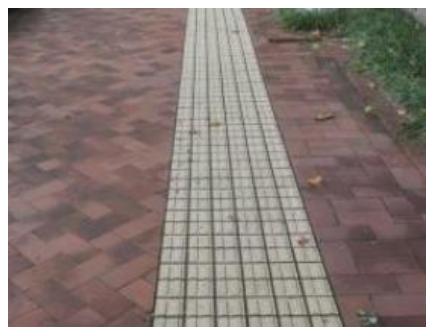

(a) ORIGINAL IMAGE

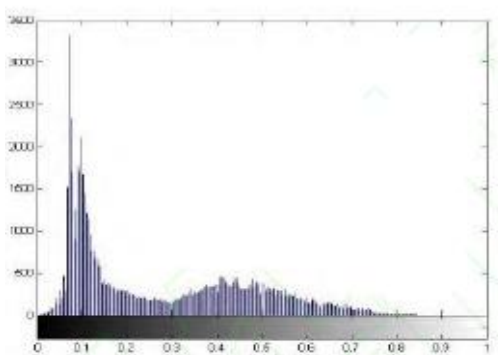

(b) HISTOGRAM OF SATURATION
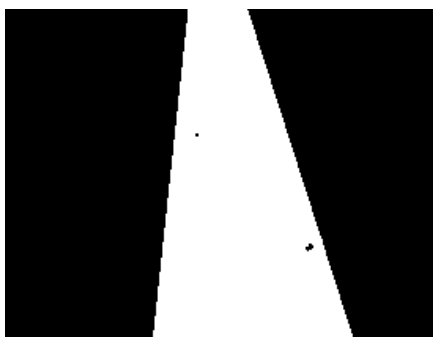

(c) THE SEGMENT IMAGE

FIGURE V. RESULT OF IMAGE SEGMENTATION

However, sometimes the color histogram will appear "jagged" shape. It is difficult to divide the image through the peaks and troughs in the histogram. So in order to determine the segmentation point, first use the Gauss smoothing filter to smooth the original color histogram. Suppose $\mathrm{S}_{\mathrm{L}}(\mathrm{x})$ is the histogram of saturation. The processed color histogram function is:

$$
\mathrm{F}(\mathrm{x}, \sigma)=\mathrm{S}_{\mathrm{L}}(\mathrm{x}) * \mathrm{G}(\mathrm{x}, \sigma)
$$

Here, $\mathrm{G}(\mathrm{x}, \sigma)$ is the Gauss function. * presents convolution.This operation not only reduces noise, but also eliminates some tiny saw teeth. 


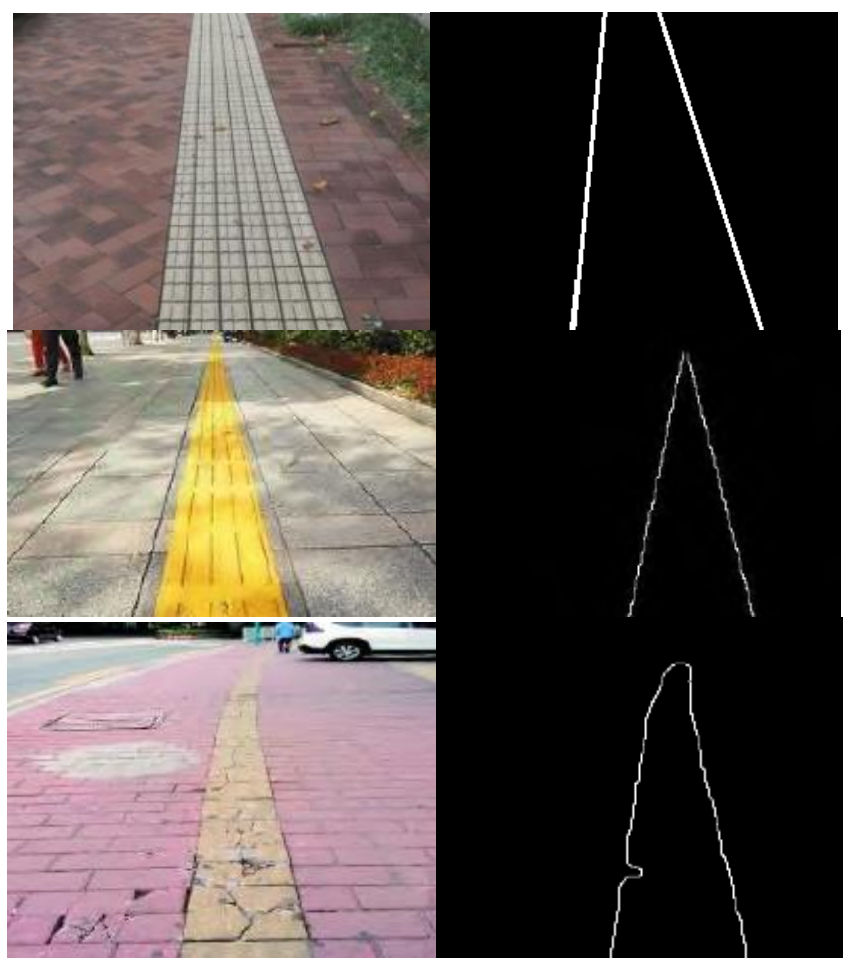

FIGURE VI. RESULT OF BLIND ROAD RECOGNITION

\section{GPS RECOGNITION}

The remote positioning function can send the blind people to the blind's relatives and friends in real time, strengthen the connection between the blind and relatives and friends, and ensure the safety of the blind. Using SIM 808 module and SIM telephone card to realize GPS remote positioning. Through the GPRS flow data of SIM 808 GPS upload module programming through the SIM card to the China Mobile networking platform, open platform (OneNET) by computer through the GPS data from the OneNET platform and processed, displayed on the map to achieve GPS remote location positioning module.

\section{CONCLUSIONS}

Aiming at the needs of the blind, a guide system is designed. According to the confirmation of the blind activities restrict the function of the system and established the general scheme of the robot; then the realization of recognition, such as the zebra and blind important traffic signs. However, the system needs to be further improved, then to realize traffic lights recognition, intelligent guide systemdevelopment.

\section{REFERENCES}

[1] Meng Xiangwei, Yan Xijun, Ouyang, stars, et al. Design of guide bar based on ultrasonic sensors [J]. electronic design engineering, 2012, 20 (17): 11-14.

[2] Zhang Zhimei, Cheng Liying, Zhao Yiheng, et al. Study of guide robot based on fuzzy PID control algorithm [J]. Journal of Shenyang Normal University (NATURAL SCIENCE EDITION), 2015,33 (1): 81-85.

[3] Tang Zhichao, Su Lin, He Chao, et al. Research on traffic sign visual recognition technology of guide robot [J]. computer technology and development, 2014 (9): 23-27.

[4] Yang Wanhai. Multisensor data fusion and its application [M]. Northwest University of Electronic Science and Technology Press, 2004.
[5] Xu Yan, Wei Zhen Yu. An improved traffic sign image recognition algorithm [J]. laser and Optoelectronics Progress, 2017 (2): 118-125.

[6] Khatib O. Real-Time Obstacle Avoidance for Manipulators and Mobile Robots[C]// IEEE International Conference on Robotics and Automation Proceedings. IEEE, 1986:500-505.

[7] Huang Yanbiao, Luo Guangyue, ho ho. Application of BP neural network in multisensor dat a fusion of patrol robot [J]. Journal of sensing technology, 2016, 29(12): 1936-1940.

[8] Otsu N. A Threshold Selection Method from Gray-Level Histograms [J]. IEEE Transactions on Systems Man \& Cybernetics, 2007, 9(1):62-66. 